\title{
ATTENUATION OF BLAST EFFECTS FROM UNDERWATER EXPLOSIONS IN BUILT STRUCTURES
}

\author{
CHRISTOPH ROLLER \& ALEXANDER STOLZ \\ Fraunhofer-Institute for High-Speed Dynamics, Ernst-Mach-Institut (EMI), Germany
}

\begin{abstract}
The paper provides reassessment and new design of shock-absorbing retrofit systems for water-filled ducts based on experimental investigations. In a first step, tests were conducted in a 2.0-meter-high circular cylinder tank made of steel. The axisymmetric geometry of the tank offered an ideal environment for validation of the theoretical work. A number of parameter variations (water level, charge location, retrofit system) provided further insight into potential and limitations of the concepts. In a second step, a reinforced concrete shaft plus relief pool will used for scaled experiments. This will serve for a realistic representation of the phenomena in a water duct and thus enabled investigations of more complex processes (multiple reflections, tunnel effect) and practical proof of concept. Regarding retrofit systems, three concepts have been identified with respect to their impedances mismatch with water: a single layer concept made of polyvinyl chloride (PVC) foam and polyurethane (PUR) foam respectively as well as a bubble curtain. The significant potential of all investigated measures has been confirmed during the test campaign. Maximum reduction of peak-overpressure down to $0.3 \%$ for PVC, $3.2 \%$ for PUR and $1.6 \%$ for the bubble curtain compared to unmitigated set-up (100\%) have been determined. Thus, the developed concepts have been successfully validated and may be applied to all areas where safety and security of structures filled with or covered by fluids. Examples include among others power plants, chemical industry, port facilities and canal constructions.

Keywords: underwater explosion, fluid-structure interaction, protective design, shock-absorption.
\end{abstract}

\section{INTRODUCTION}

The importance of critical infrastructure protection did increasingly grow within the last decades due to numerous extreme loading scenarios resulting from natural disasters, accidents and sabotage (i.e. Fukushima 2011, Tianjin 2015). Although safety and security had always been addressed in the field of industrial and power plant design the increased concern about security requires a critical review of existing and well-established methods. Effective measures for attenuation of shock waves at explosions in coolant ducts represent one sensitive aspect within this context.

The danger of underwater explosions does come from the fact that pressure waves are transmitted very effectively from the fluid to submerged or surrounding structures [1]. Furthermore, even high amplitude waves lie in an acoustic range where natural dissipation is small due to the lack of non-linearity of the material. This is particularly important when there is no or negligible geometric dissipation, as is the case with wave propagation in a constant cross-section channel. In such cross-sections, waves can propagate widely without significant energy reduction. In fact, in most cases the observable reduction of energy is due to the structural response (deformation of the channel walls) of the environment itself.

An up-to-date overview of attenuation of explosive effects can be found in [2], which also considers underwater explosions. In general, there are two approaches to achieve the desired reduction and thus the desired protection: the first approach is based on the arrangement of lowered, fixed barriers. These serve as refractive bodies and can in principle reduce both significant input parameters of peak pressure and momentum (pressure-time-integral). To obtain a high damping effect, the material of the barrier should have a high impedance (as a result of high density and stiffness) compared to the fluid since otherwise the wave will be 
largely transmitted through the barrier instead of being broken. This boundary condition severely restricts the achievable effectiveness in underwater explosions in practice. Furthermore, barriers in channels are impracticable because they block the cross section. An alternative approach involves lowered, deformable objects which, when compressed by interaction with the shaft, reduce the pressure due to the associated expansion of the fluid. This approach can be implemented in several ways: bubble-like curtains, floating gas-filled containers or deformable wall panels [3] are examples here. Such elements allow a high reduction in peak pressure, but generally only a slight reduction in momentum, since only relatively small forces are exerted on the fluid. The reduction of the peak pressure is thus accompanied by the increase of the wavelength. Devices for damping and their interpretation by means of numerical simulation methods continue to be the subject of current research and found in the literature in the field of various applications, such as square honeycomb structures [4], honeycomb structure with rubber pad [5] for the protection of ship structures, matrix of aluminium tubes [6], air-filled structures [7] and the effect of air bubbles [8].

According to current practice and state of research, two application types with big potential have been chosen for experimental analysis regarding their efficiency towards reducing pressure waves in ducts. Test environment plus related analysis of the single application types will be presented in the following.

\section{ANALYSIS OF POTENTIAL MITIGATION METHODS}

Two kinds of test specimen have been defined for experimental analysis. On the one hand tests have been conducted in a circular cylinder barrel made of steel. This specimen provides the option to do a number of parameter investigations (water level, charge location, bedding influence) at minimum effort in planning and time. Another advantage is the good possibility of validation due to the axisymmetric geometry. On the other hand, a reinforced concrete tunnel plus relief pool will be used for scaled experiments in future for a realistic representation of the phenomena in a water.

\subsection{Experimental setup of cylindrical steel barrel}

The experimental setup with a cylindrical steel barrel is outlined in Fig. 1. The circular crosssection has an inner diameter of $1.0 \mathrm{~m}$ with a wall thickness of $1.0 \mathrm{~cm}$.

Piezoelectric pressure sensors were arranged right across the diameter at a charge height of $1.0 \mathrm{~m}$ and $0.5 \mathrm{~m}$ above the cylinder bottom, respectively. Thus, two redundant
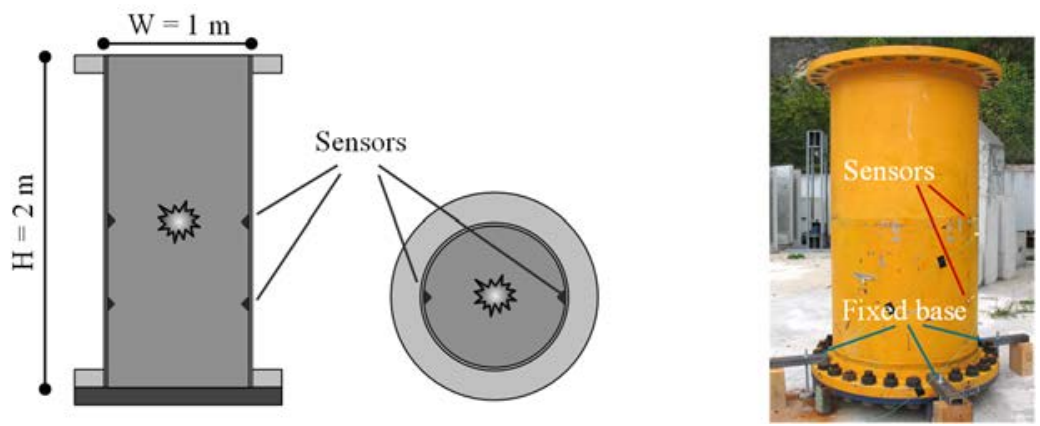

Figure 1: Illustration of cylindrical steel barrel, sketch in longitudinal- and cross-section (left) and on test site (right). 
measurement signals could be recorded for each altitude per trial. Each configuration was repeated twice for taking into account the experimental scattering.

The configuration of a completely filled barrel, water column of $2.0 \mathrm{~m}$, represents the reference configuration without any mitigation methods installed. Fig. 2 illustrates the overpressure recordings at height of charge for the two sensors installed across the diameter for the first $5.0 \mathrm{~ms}$. The first shock wave with a peak-overpressure of about $28 \mathrm{MPa}$ is followed by cavitation phenomena with minor negative overpressure and a second pressure wave from reflection at the barrel wall with an increased load duration. This characteristic shape was detected for all repetitions of this configuration.

\subsection{Mitigation methods}

Two approaches for mitigation have been chosen for investigation: a bubble curtain and a single layer concept made of foam. For the latter approach two materials have been chosen with respect to their impedance mismatch with water: polyvinyl chloride (PVC) foam and polyurethane (PUR) foam. Fig. 2 displays the installed mitigations methods.

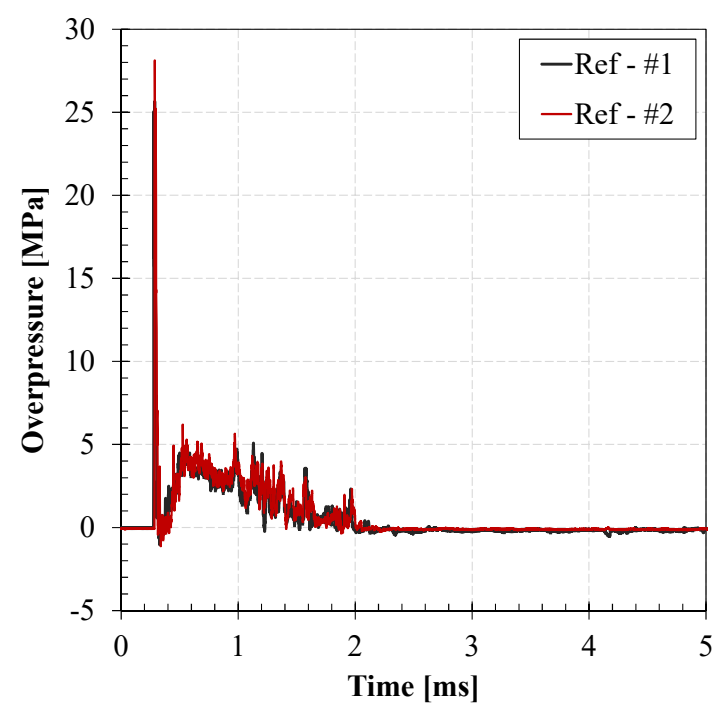

Figure 2: Overpressure time history of reference test without mitigation measure for sensor \#1 and \#2 at height of charge.
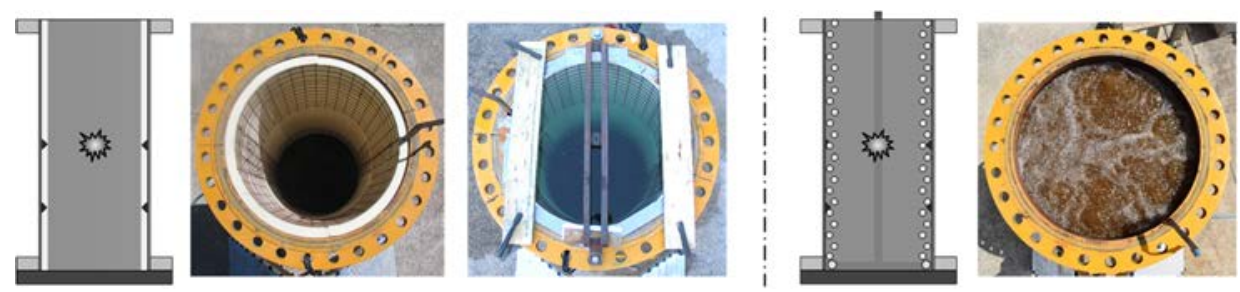

Figure 3: Illustration of various tested mitigation methods. 


\subsubsection{PVC - status quo}

In the area of coolant ducts PVC based foam elements have been identified as most frequently used mitigation measure according to concurring statements from plant operators. The durability of these elements has been investigated with special interest on functionality at different points of service life. Evaluation of existing static test results prove a significant degradation of the element's effectiveness when applied at continuous operation [9]. An average loss of one quarter of material thickness over a duration of 32 months due to diffusion related outgassing under hydrostatic pressure (no loss in weight) resulted in an increase of material density. The related increase in material impedance consequently lead to lack of performance with regard to mitigation of shock wave loading. This property makes this material maintenance prone and thus uneconomical for permanent use. Since the PVC based solutions still represents the status quo it is investigated in the present study for comparison purpose.

Fig. 4 illustrates the resulting overpressure-time histories for the sensors at height of charge for two out of three tests (highlighted in green). The additional black curve represents the average history of theses curves. Compared to the reference case displayed in Fig. 2 the attenuation effect for the pressure values is clearly visible. While the first peak-overpressure is completely eliminated the second peak-overpressure is reduced to value of $0.15 \mathrm{MPa}$. Thus, the momentum has been mitigated, too, although the load duration increased by factors of more than 10 .

\subsubsection{PUR - alternative foam material}

The evaluation of an alternative wall lining using polyurethane foam elements is presented in the following. The material is qualified for damping layer application due to its low density as well as its good elastic-plastic behaviour that is a distinctive plateau in the stress-strain

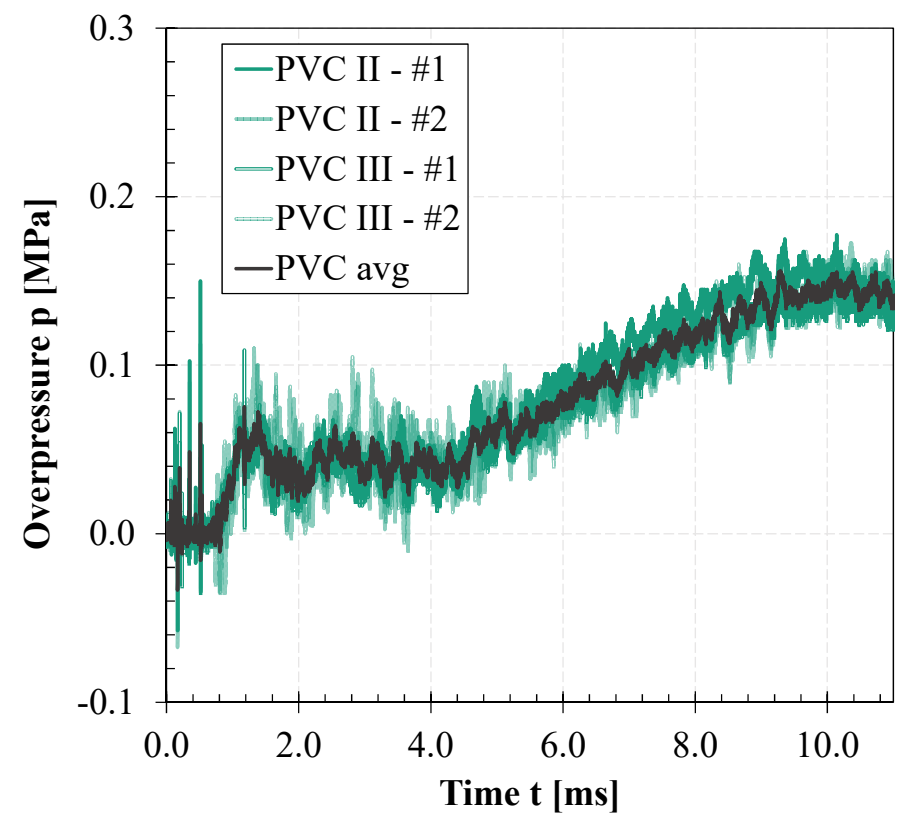

Figure 4: Overpressure time history of test no. II and III with PVC coating for sensor \#1 and $\# 2$ at height of charge plus average regime. 
history. The PUR foam was investigated in two density classes, on the one hand with $50 \mathrm{~kg} / \mathrm{m}^{3}$ at the lower bound and on the other hand with $200 \mathrm{~kg} / \mathrm{m}^{3}$ at the upper bound of the material production. Since the density, as an impedance characteristic, directly enters into the reflection process during the fluid-structure interaction, a maximum factor of 4 in pressure reduction between the selected samples has been expected. Fig. 5 displays the averaged blast parameters for the two PUR samples and proves the theoretical prediction. The measured values of peak-overpressure amount to $2.4 \mathrm{MPa}$ and $0.8 \mathrm{MPa}$ for the samples with higher and lower density, respectively. The actual attenuation capacity does rely on the amount of water being soaked in by the liner material before the blast load was initiated. Thus, the theoretical assumption based on purely dry material is violated even though the water absorption should not have exceeded $3.0 \%$.

Comparing the doted impulse histories, the difference between the two samples is rather small. This results from a decreased load duration for the high-density material compensating the initially higher-pressure values. With respect to the reference case without mitigation measure this alternative foam material still leads to a significant decrease in loading for the surrounding structure.

\subsubsection{Bubble curtain - alternative method}

Starting from the reference case, the supply of compressed air and thus the bubble fraction was varied while maintaining the charge location and quantity. All configurations with compressed air were repeated twice just as the all the trials of the test campaign before. Fig. 6 displays the determined load parameters over time for the bubble curtain concept and underlines the potential of the concept. Reason for the efficient attenuation is the huge

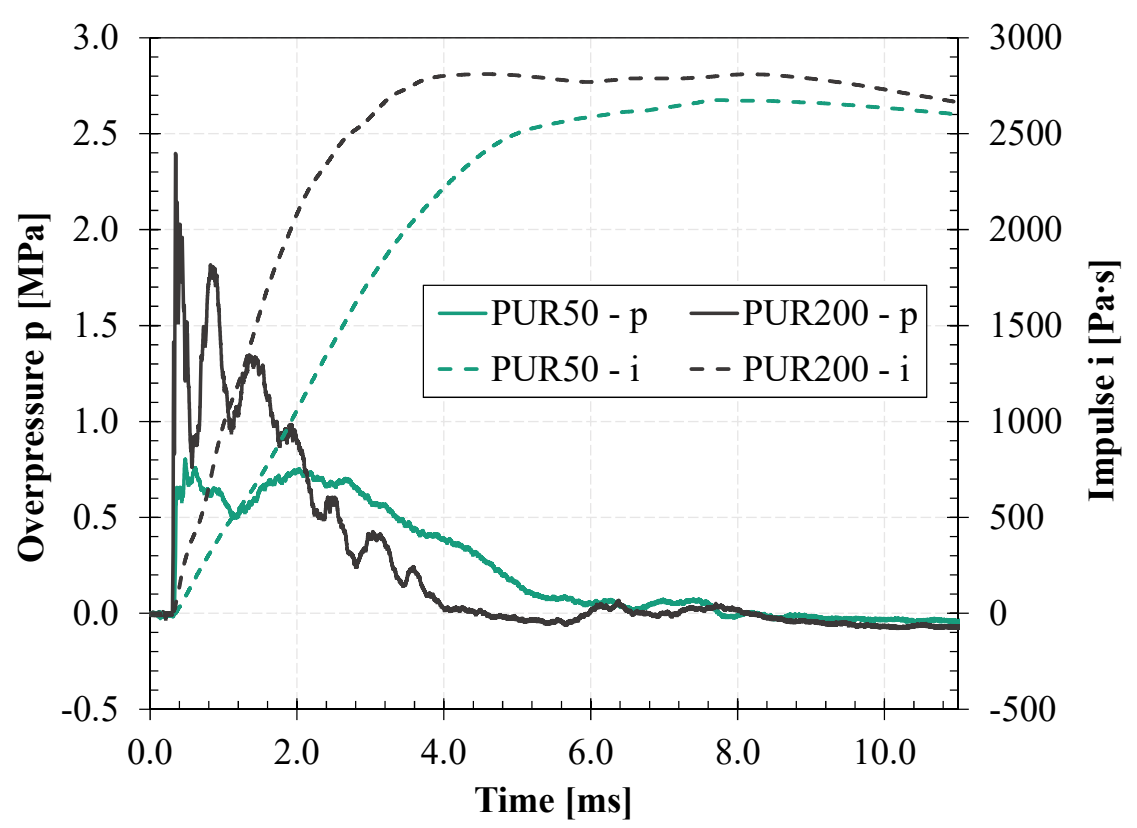

Figure 5: Average overpressure and impulse time history of tests with PUR foam with densities of 50 and $200 \mathrm{~kg} / \mathrm{m}^{3}$. 


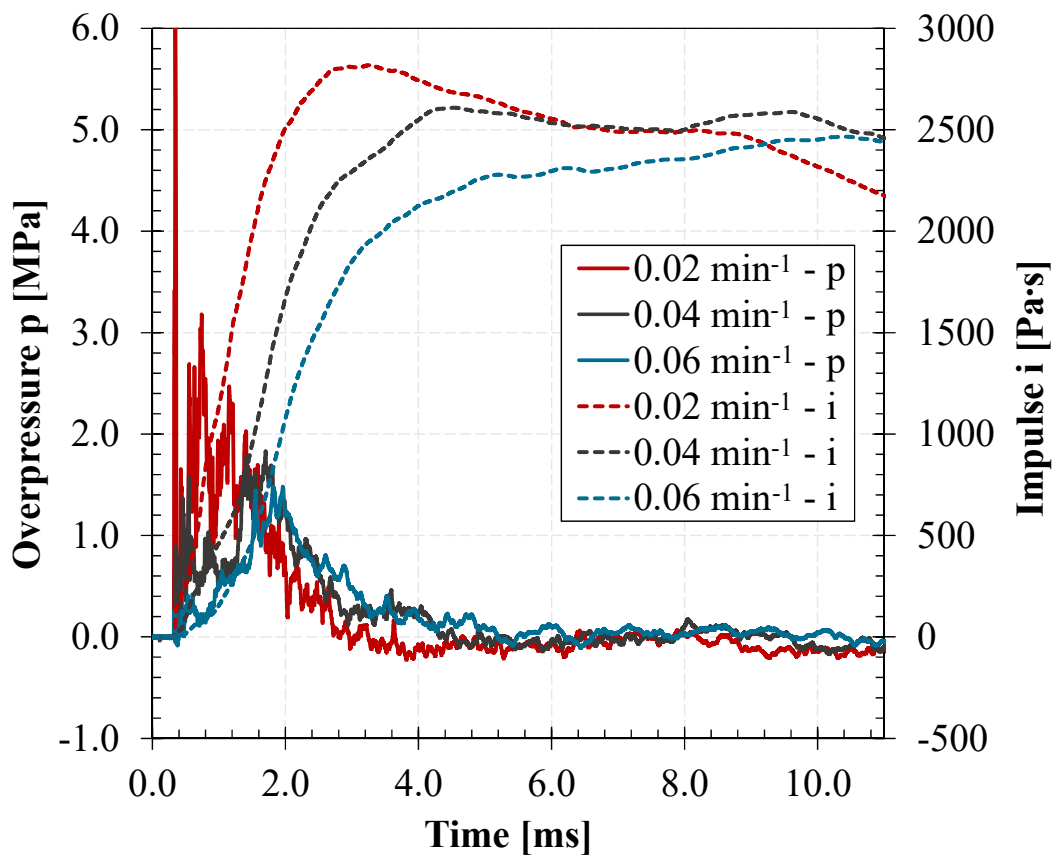

Figure 6: Average overpressure- and impulse-time history of tests with bubble curtain of air supply between 0.02 and $00.6 \mathrm{~min}^{-1}\left(\mathrm{~m}^{3}\right.$ air per $\mathrm{m}^{3}$ water per minute).

difference in impedance between water and air on the one hand. On the other hand, generation of a big amount of surface area by the single bubbles directly contributes to the wave fracture process. Effectively, the peak-overpressure could be reduced by approx. $75 \%$ even with low compressed air supply of $0.5 \mathrm{~cm}^{3} / \mathrm{ms}$ or $0.02 \mathrm{~m}^{3}$ air per $\mathrm{m}^{3}$ of water per minute. Increasing the supply by a further $1.0 \mathrm{~cm}^{3} / \mathrm{ms}$ or $0.04 \mathrm{~min}^{-1}$ even reduces the peak pressure to less than $1.7 \%$ of the reference value without bladder curtain. The doted lines show the influence on the resulting impulse load. The impulse-time profile, which is averaged out of every three experiments, is also plotted as a function of the volume of air supplied per minute per existing volume of water. In contrast to the peak pressure here, the impact damping levels almost independent of the supplied air volume. The reductions converge at around $30 \%$.

\subsubsection{Comparison of investigated methods}

Fig. 7 gives comparatively evaluates the investigated mitigation measures by means of degree of attenuation for the decisive load parameters. The degree of attenuation is normalized with respect to the reference case without any mitigation measure applied. This means for the bubble curtain with an air supply of $0.02 \mathrm{~m}_{\text {air }}{ }^{3} / \mathrm{m}_{\text {water }}{ }^{3}$ per minute that the resulting first peakoverpressure is only $25 \%$ of the reference case.

Generally, it can be stated that bubble curtain and PUR foam have a similar performance with further potential depending on the chosen configuration. Values of first and second peak-overpressure can be mitigated significantly while the momentum remains in a range of $70 \%$ compared to the reference case. The PVC foam delivers outstanding attenuation for all load parameters. If there were no durability and thus maintenance issues in first place, it would be the measure of choice. 


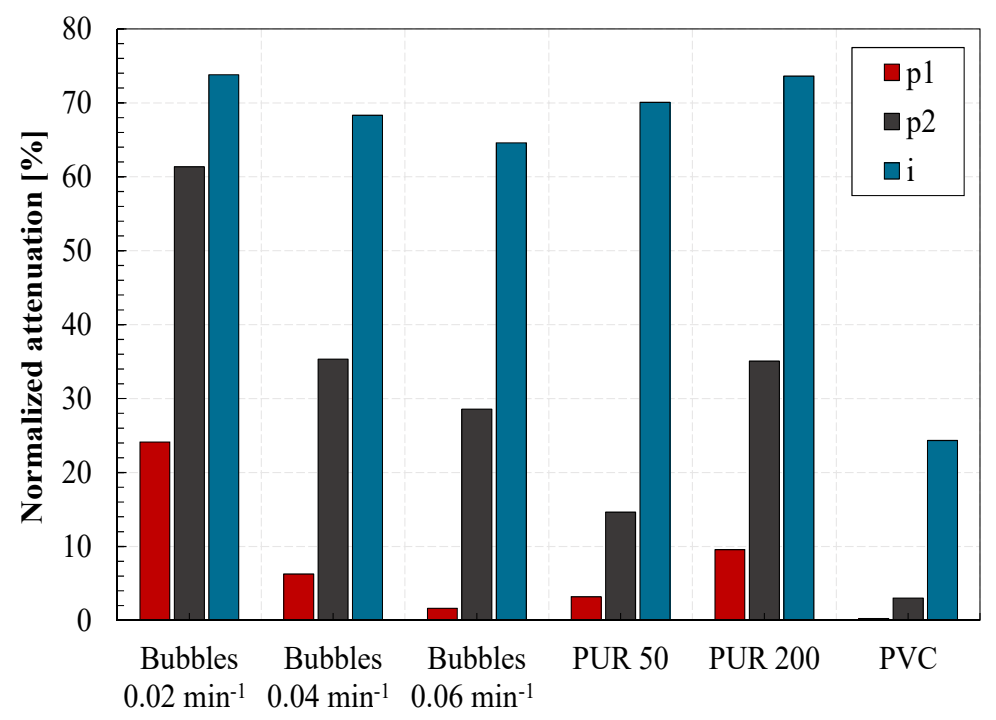

Attenuation measure

Figure 7: Comparison of efficiency of applied methods for first peak-overpressure p1, second peak-overpressure $\mathrm{p} 2$ and impulse $\mathrm{i}$.

\section{SUMMARY AND OUTLOOK}

For the purpose of retrofitting coolant ducts with respect to mitigation of underwater explosion effects, current practice and alternative application have been analysed experimentally. All of the mitigation measured were able to significantly decrease the shock loading.

In the next step, a reinforced concrete shaft plus relief pool will used for model-scaled experiments. This will serve for a realistic representation of the phenomena in a water duct. The future investigations will provide more insight into more complex processes (multiple reflections, tunnel effect) and practical proof of concept.

\section{REFERENCES}

[1] Cole, R.H., Underwater Explosions, Dover Publ., New York, 1948.

[2] Shukla, A., Rajapakse, Y.D.S. \& Hynes, M.E., Blast Mitigation: Experimental and Numerical Studies, Springer, 2013.

[3] Klomfass, A., Neuwald, P. \& Thoma, K., Attenuation of underwater blast waves in ducts by air-filled wall panels. Proceedings of 23rd Int. Symp. of Shock Waves, ed. F. Lu, Texas, 2001.

[4] Wadley, N.G. et al., Dynamic compression of square honeycomb structures during underwater impulsive loading. Journal of Mechanics of Materials and Structures, 2(10), pp. 2024-2048, 2007.

[5] Chen, Y., Tong, Z.P., Hua, H.X., Wang, Y. \& Gou, H.Y., Experimental investigation on the dynamic response of scaled ship model with rubber sandwich coatings subjected to underwater explosion. Int. Journal of Impact Engineering, 26(2), pp. 318-328, 2008.

[6] Glascoe, L.G., Margraf, J., McMichael, L. \& Vandersall K.S., A mitigation scheme for underwater blast: Experiments and modeling. Proc. Of AIP Conf., 1426(1), pp. 721724, 2012. 
36 Structures Under Shock and Impact XV

[7] Glascoe, L.G., Margraf, J., McMichael, L. \& Vandersall K.S., Underwater blast experiments and modeling for shock mitigation. 14th Int. Detonation Symp. Coeur D'alene, ID, United States, 2010.

[8] Lu, T., Samulyak, R. \& Glimm, J., Direct numerical simulation of bubbly flows and application to cavitation mitigation. Journal of Fluids Engineering, 129(5), pp. 595604, 2006.

[9] N.N., Druckversuche an PVC-Schaumstoffen/Prüfbericht, MPA Stuttgart, 1994. 